\title{
Neuromarkers of anxiety and depression in a patient after neuro-ophthalmic surgery of the meningioma - effect of individually-tailored tDCS and neurofeedback
}

\author{
Andrzej Mirski ${ }^{1}$, Maria Pąchalska, ${ }^{1,2}$, Marek Moskała ${ }^{3}$, Michał Orski ${ }^{4}$, Małgorzata Orska ${ }^{4}$, \\ Maria Miąskiewicz ${ }^{4}$, Jan Zapała ${ }^{5}$, Juri D. Kropotov ${ }^{6,7}$ \\ ${ }^{1}$ Chair of Neuropsychology, Andrzej Frycz Modrzewski University, Cracow, Poland \\ ${ }^{2}$ Center for Cognition and Communication, New York, NY, USA \\ ${ }^{3}$ Department of Neurosurgery and Neurotraumatology, CMUJ, Cracow, Poland \\ ${ }^{4}$ Department of Ophtalmology, Ludwik Rydygier Memorial Specialist Hospital, Cracow, Poland \\ ${ }^{5}$ Maxillofacial Surgery Department, Ludwik Rydygier Memorial Specialist Hospital, Cracow, Poland \\ ${ }^{6}$ Laboratory for theNeurobiology of Action Programming, Institute of the Human Brain, Russian Academy of Sciences, St. \\ Petersburg, Russia \\ ${ }^{7}$ Institute of Psychology, Norwegian University for Science and Technology, Trondheim, Norway
}

Mirski A, Pąchalska M, Moskała M, Orski M, Orska M, Miąskiewicz M, Zapała J, Kropotov JD. Neuromarkers of anxiety and depression in a patient after neuro-ophthalmic surgery of the meningioma - effect of individually-tailored tDCS and neurofeedback. Ann Agric Environ Med. 2015; 22(4): 718-723. doi: 10.5604/12321966.1185783

\begin{abstract}
The aim of the study was to evaluate the effectiveness of individually tailored anodal tDCs/ neurofeedback protocol for the reduction of post-operative depression after a neuroophtalmological operation of the meningioma. The neuromarkers in Quantitative EEG (QEEG) and Event-related potentials (ERPs) were utilized in the construction of protocol and evaluation. Case description. A 45-year-old female after successful neuro-ophthalmic surgery of the meningioma, complained of severe pain and anxiety, difficulties with sleeping, attention and memory problems, as well as inability to continue working in her given profession. Neuropsychological testing showed lack of cognitive disturbances and post-operative depression. Two working hypotheses were tested to find neuromarkers of depression and anxiety. In line with the 'depression hypothesis' a frontal alpha asymmetry pattern was found in the patient, and in line with the 'anxiety' hypothesis an increased left temporal P1 wave in response to visual stimuli was found in ERPs. A specific alpha asymmetry neurofeedback protocol combined with an anodal tDCS was suggested. Twenty sessions of individually-tailored anodal tDCs/ neurofeedback protocol were performed. The QEEG frontal asymmetry pattern and the excessive temporal P1 wave were normalized after the intervention. Conclusions. The patient recovered from post-operative depression and returned to work after 20 sessions of the combined neurofeedback/tDCS protocol. Specific patterns of QEEG and ERPs serve as neuromarkers for constructing the protocol and for monitoring the results of intervention.
\end{abstract}

\section{Key words}

chronic pain, post-operative depression, pain

\section{INTRODUCTION}

After undergoing any kind of major surgery, there may be many side-effects, not least of which is depression that can make the patient's recovery that much more excruciating [1]. Although this aspect of surgery is not often given the attention it deserves, it is a very real possibility for those faced with a serious operation. Coupled with the fact that most surgeons are mostly concerned only with the patient's physical recovery, rather than their mental status, such a post-operative depression may linger longer than is necessary, further prolonging the recovery process. It is not exactly what factors cause the depression, although there are some thoughts are taken into consideration. As depression may

address for correspondence: Maria Pąchalska, Chair of Neuropsychology, Andrzej Frycz Modrzewski Krakow, University, Herlinga-Grudzinskiego 1, 30-705 Kraków, Poland

E-mail: neuropsycholgia23@o2.pl

Received: 20 July 2015; accepted: 30 September 2015 strike any surgery patient, there are theories about exactly which aspects of the surgery may have a detrimental effect on patients, such as anesthesia from the surgery, antibiotics and other medications given to treat pain, disorientation after the surgery, digestive problems caused by medication given to the patient, Post-Surgical Traumatic Stress Syndrome, soreness and pain, and being bedridden in the recovery process [2].

The need for surgery may vary according to the seriousness of the diagnosis. A more serious diagnosis brings with it a more severe emotional reaction [3]. For example, is common for people facing ophthalmic surgery, to feel extremely anxious and depressed about the outcome [4]. The same is true for an individual facing surgery for the removal of a cancerous tumour $[1,5,6]$. The site of the tumour is not without significance. If this is the face, or surroundings of the eye, emotional reaction can be stronger, because it is a place of greater cortical representation [1]. Therefore, it is understandable that depression can occur in a patient after neuro-ophthalmic surgery for meningioma. Even if it is not be a malignant tumour, the location and 
consequences of proliferation are devastating, and affect the human consciousness and emotional reactions. Both the onset of severe pain and post-operative depression can expected. Because the cause of this depression is not fully understood it would be difficult to carry out effective diagnosis $[6,7]$ and treatment of people who have fallen ill. New neurotechnologies may be promising and useful in the diagnosis and treatment of these symptoms [8].

Objective. The aim of the presented study was to evaluate the effectiveness of neurotherapy for a 45 -year-old female diagnosed with post-operative depression after neuroophthalmic surgery of the meningioma. It was hypothesized that there would be good response to relative beta training applied to regulate the dynamics of brain function.

\section{CASE STUDY}

The patient, a 45-year-old female, noticed in January 2014, oedema of the eyelids of the left eye. In July 2014, there occurred partial disturbance to sight, in particular when the patient was looking downwards, and a temporary paralysis of the left side of the face.

$\mathrm{CT}$ and MRI showed a tumour covering the bone structures of the left eye socket (the lateral wall) and infiltration into the base of the middle cranial fossa. A neurological test conducted upon admission did not confirm any focal symptoms of brain damage. In ophthalmological testing, the left eye globe showed limited exophthalmos. Anterior and posterior segments were within normal limits. The left eye ball was set with axial exophthalmos (right eye $14 \mathrm{~mm}$; left eye $18 \mathrm{~mm}$; base $100 \mathrm{~m}$ ) without any limitations in mobility, with good distance and reading vision. Visual field for both eyes was normal. Intraocular pressure in the right eye was 12 $\mathrm{mmHg}$, in the left eye $-13 \mathrm{mmHg}$. The patient was operated on in an interdisciplinary unit by a neurosurgeon and a maxillofacial surgeon. Through 'lazy S' access by means of lateral orbitotomy a part of the tumour removed was removed from the eye socket, and material was taken for histopathological testing. The patient reacted well to the procedure. After the procedure, the patient was awakened and was in verbal contact; however, there was confirmed an impairment to the mobility of the left eye ball within the scope of abduction and adduction with double vision. This was mobilised and independent. After a control TK of the head had been carried out the patient was discharged home.

In the resulting TK testing (the state following the removal of the left eye socket tum removed was our), partially ascertained was sclerotically removed reconstructed bone tissue derived from the great wing of the sphenoid bone bulging in the area of the temporal muscles. In addition similarly: a still visible osteosclerosis reconstruction in the area of the great wing of the sphenoid bone on the left side bulging into the eye socket to around $7 \mathrm{~mm}$ modelling and relocating medially the ordinary lateral muscle, narrowing the superior orbital fissure, as well as narrowing the orbital apex. The changes bulge to the middle cranial fossa on the level of the pole of the temple lobe, with segmentally visible calcification in the topography of the meninges. Within the brain the shading of the brain tissue was normal. The cell system was of normal width, without translocation. The subarachnoid expanse did not widen.
Histopathological testing confirmed Meningeoma meningotheliale $\mathrm{WHO} 1$ penetrating through the bone.

Several days after the procedure there occurred pain engulfing the eye, as well as headaches, which intensified particularly in the evening. In the ophthalmolgical tests conducted after the operation, no visible pathology in the anterior and posterior segment was noted. The left eye globe was positioned in a slight exophthalmos (right eye $14 \mathrm{~mm}$; left $16 \mathrm{~mm}$ ). Maps of nerve fibres and GCC complexes did not showed any pathology (Fig. 1). The visual field was normal for both eyes - carried out using 2010 Carl Zeiss Meditec Humphrey Visual Field Analyzer. Optical coherence tomography (Optovue RTVue OCT) did not show any significant changes in morphology of the macula and nerve fiber layer (Fig. 2 and 3).

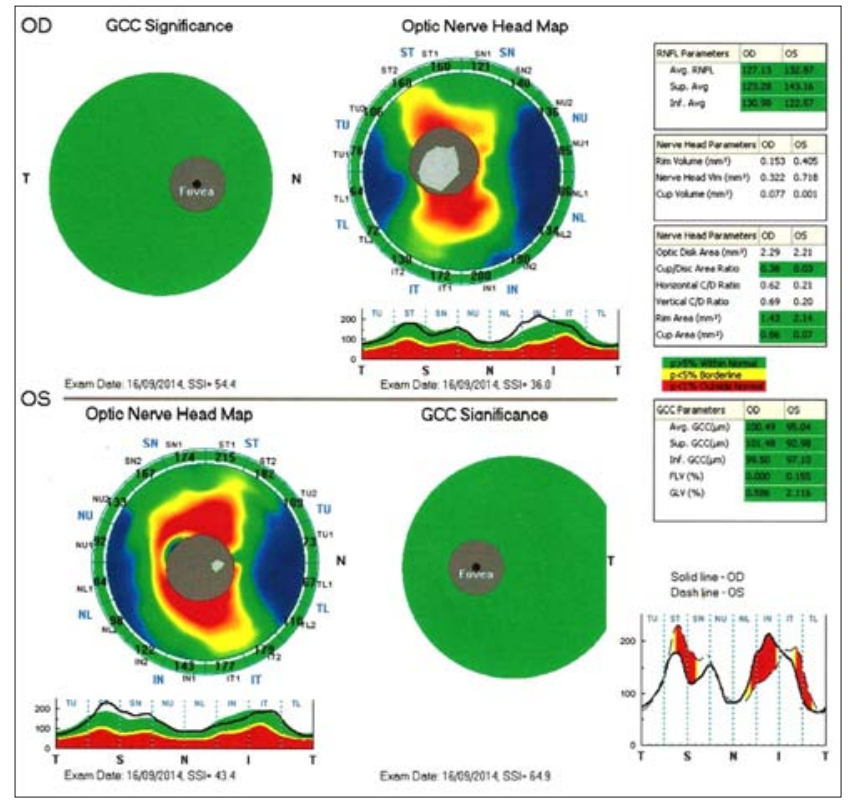

Figure 1. Maps of nerve fibres and GCC complexes

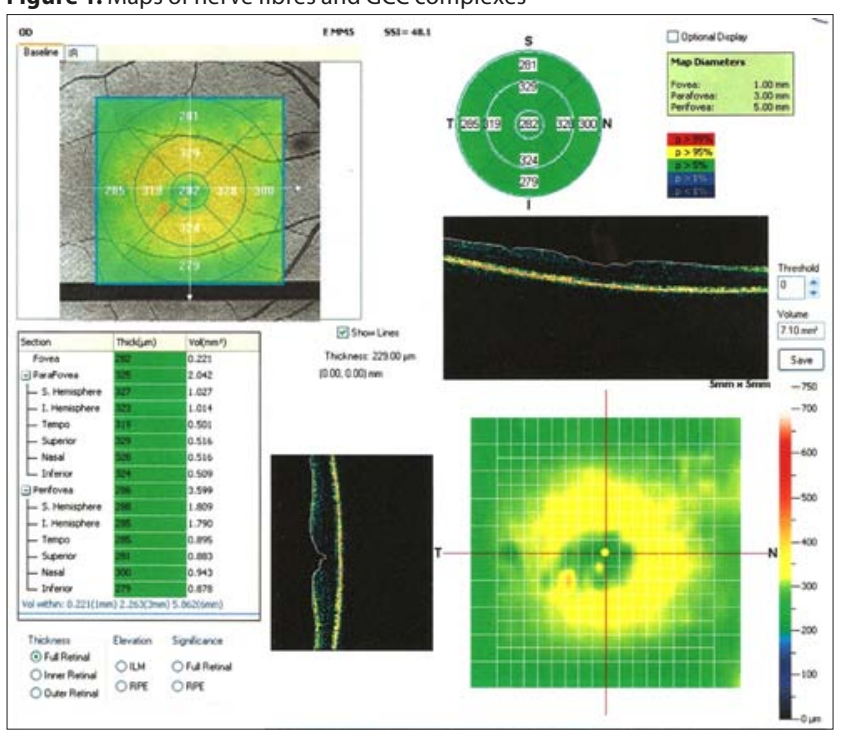

Figure 2. Macula morphology of the right eye

One week after surgery the patient started to feel that the worst has passed: she was able to shower and dress herself, and began the process of returning to some form of normal life. One month later, however, the patient complained of 


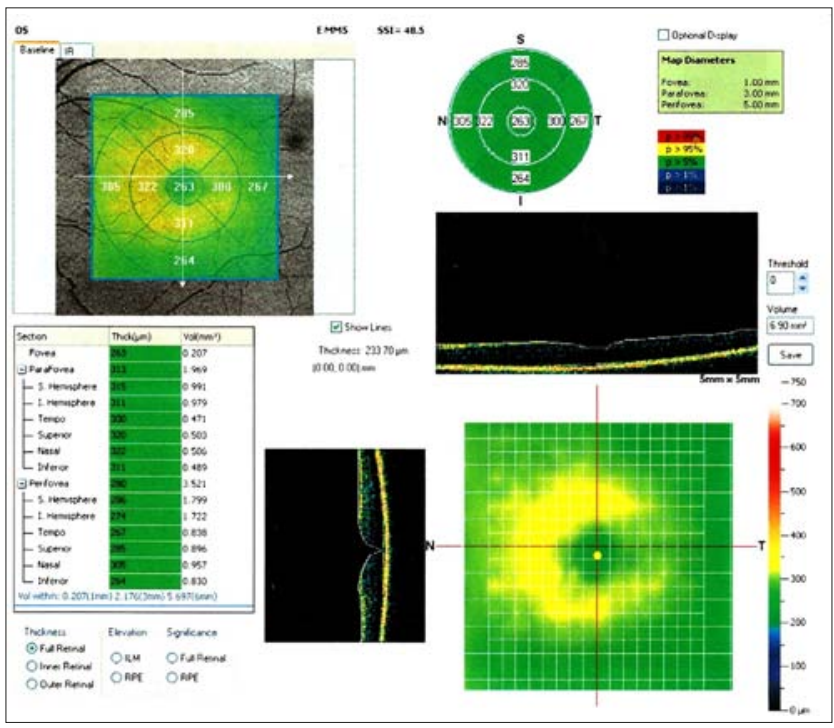

Figure 3. Macula morphology of the left eye

increasing occurrences of difficulties with sleeping resulting from anxiety and a constant intense spike in pain despite taking painkillers. When informed by the neurosurgeon that at least one more major surgery would be necessary in the next year, she began to become obsessed about it. She also complained that her memory was getting worse. After six months, the patient complained of severe pain and anxiety, difficulties with sleeping, attention and memory problems, as well as an inability to continue work in her given profession. Her life, as she put it, 'began to look like one painful and sad way into the grave.' In her opinion, she experience post-surgical depression because it forced her. to confront her own mortality.' What made it especially difficult and dangerous was that she never felt depressed or sad before the surgery.

Neuropsychological testing. In the neuropsychological testing utilising the Polish version of the WMS-III Memory Test [2], neither in the first test (pre-operation) nor in the second test (post-operation) were disturbances found in the dynamics of memory, logical memory and spatial-visual memory, both in instant reproduction and in delayed. No disturbances in attention were noted.

In the Beck Depression Scale administered several times during the whole treatment procedure (cf. Fig. 4), the patient was not depressed before surgery (10 pts). One month after surgery, she showed mild depression (20 pts), and 6 months after surgery severe depression (32 pts).

The patient took part in 20 sessions of individuallytailored anodal tDCs/ neurofeedback therapy twice a week. The neuromarkers in Quantitative EEG (QEEG) and Event related potentials (ERPs) were used in the construction of protocol and evaluation.

After the neurotherapy, the patient obtained a score of 6 points on the Beck Depression Scale, while after 6 months of follow-up a score of 7 points. These results mean that the symptoms of post-surgical depression had passed. She no longer complained of chronic pain, started to sleep better and perform everyday duties and tasks. She returned to her work as a professional teacher, with which she coped splendidly. She claimed that she was able to take pleasure again in her life.

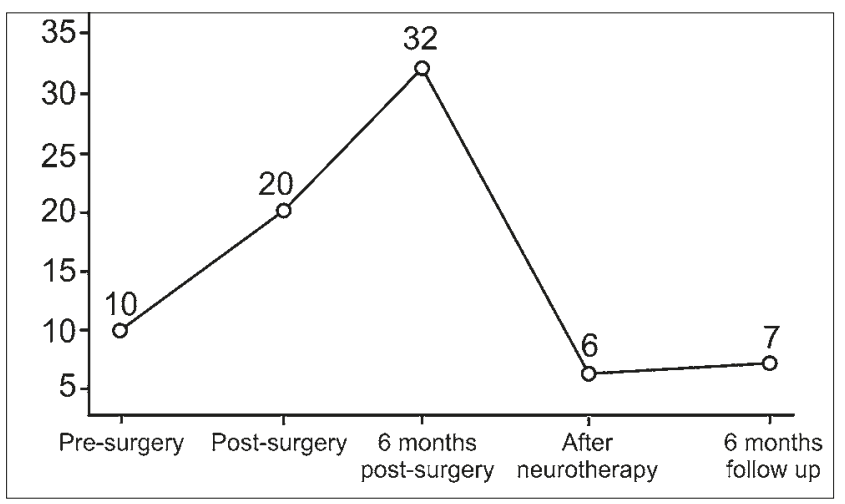

Figure 4. Results obtained on the Beck Depression Scale prior to the introduction of neurotherapy, after 20 sessions of neurotherapy and after 6 months of follow-up

Neurophysiological testing - EEG recording. Electroencephalogram (EEG) was recorded with a Mitsar 21-channel EEG system (Mitsar, Ltd., http://www. mitsarmedical. com), with a 19-channel electrode cap with tin electrodes that included $\mathrm{Fz}, \mathrm{Cz}, \mathrm{Pz}, \mathrm{Fp} 1 / 2, \mathrm{~F} 3 / 4, \mathrm{~F} 7 / 8$, T3/4, T5/6, C3/4, P3/4, O1/2. The Electro-cap was placed on the scalp according to the standard 10-20 system. Electrodes were referenced to linked earlobes (off-line) and the input signals were sampled at a rate of $250 \mathrm{~Hz}$ (bandpass $0.5-$ $30 \mathrm{~Hz}$ ). The ground electrode was placed on the forehead. Impedance was kept below $5 \mathrm{k} \Omega$. The patient sat upright in a comfortable chair looking at a computer screen (17 inches) 1.5 meter in front of them. All recordings were made by the author of this article. The ERP waveforms were averaged and computed off line, and trials with omission and commission errors were automatically excluded.

Behavioural task. The task consisted of 400 trials sequentially presented to the patient every 3 seconds. Three categories of visual stimuli were used: 1) 20 different images of animals referred to later as A; 2) 20 different images of plants - P;3) 20 different images of people of different professions (presented together with an artificial 'novel' sound) referred to as $\mathbf{H}$. The trials consisted of presentations of pairs of stimuli with inter-stimulus intervals of $1 \mathrm{~s}$. The duration of the stimuli presentation was $100 \mathrm{~ms}$.

Four categories of trials were used: A-A, A-P, P-P, and P-H (Fig. 1). In the trails with A-A and P-P pairs the first and the second stimuli were identical (physically the same). The trials were grouped into 4 sessions with 100 trials in each. In each session, a unique set of $5 \mathbf{A}$ stimuli, $5 \mathbf{P}$ and $5 \mathbf{H}$ stimuli was selected. Each session consisted of a pseudo-random presentation of 100 pairs of stimuli with equal probability for each category and each trial category [8].

The task was to press a button with the right hand to all A-A pairs as fast as possible, and to refrain from pressing in response to other pairs. The patients performed 10 trials without recording to see if they understood the instruction. After completing 100 trials they rested for a few minutes. Stimuli occupied about $3.8^{\circ}$ of the visual field around the middle of the screen. Visual stimuli (were selected to have) had similar 2D sizes and luminosities.

Artefact correction procedures. Eye blink artefacts were corrected by zeroing the activation curves of individual independent components corresponding to eye blinks. These components were obtained by application of Independent 
Component Analysis (ICA) to the raw EEG fragments (as described in Vigário, 1997; Jung et al., 2000). Epochs with excessive amplitude of filtered EEG and/or excessive faster and/or slower frequency activity were automatically marked and excluded from further analysis. The exclusion thresholds were set as follows: (1) $100 \mu \mathrm{V}$ for non-filtered EEG; (2) $50 \mu \mathrm{V}$ for slow waves in $0-1 \mathrm{~Hz}$ band; and (3) $35 \mu \mathrm{V}$ for fast waves filtered in the band $20-35 \mathrm{~Hz}$. In addition, the recordings were visually inspected and the remaining artefacts excluded.

EEG spectra. EEG spectra were computed for Eyes Open, Eyes Closed and the GO/NOGO task conditions separately. The artefact free fragments of EEG were divided into $4 \mathrm{sec}$ epochs with a $50 \%$ overlap. The Hanning time window was used. EEG spectra were computed for each epoch and averaged. Mean value and standard deviations for each $0.25 \mathrm{~Hz}$ bin were computed. For comparison of the EEG spectra, pre and before intervention, the t-test was used.

Working hypotheses. Because of the patient's complaints, 2 working hypotheses were tested to find neuromarkers of depression and anxiety.

1. Hypothesis of depression. According to this hypothesis, some patients with depression show excessive frontal alpha on the left side and consequently reveal a frontal alpha asymmetry. In such cases, a specific alpha asymmetry neurofeedback protocol was suggested for the treatment symptoms of depression [9]. For testing this hypothesis, EEG spectra of the patient were computed for all conditions and compared with the reference database.

2. Hypothesis of anxiety. In ERPs of patients with general anxiety, an increase of $\mathrm{P} 1$ wave in response to visual stimuli in general, and to emotionally meaningful stimuli in particular, was observed $[10,11]$. The enhancement of the visual P1 wave was interpreted as the effect of activated amygdala on early stages of visual processing.

\section{RESULTS}

Pre-treatment assessment - Behavioural parameters. Table 1 shows omission and commission errors, reaction time and reaction time variance for the first recording before the treatment. No deviations from the reference are observed during the pre-treatment session.

Table 1. Behavioural data in the cued GO/NOGO task

\begin{tabular}{lcccc}
\hline & $\begin{array}{c}\text { Omission } \\
\text { errors }\end{array}$ & $\begin{array}{c}\text { Commission } \\
\text { errors }\end{array}$ & $\begin{array}{c}\text { Reaction time } \\
\text { (RT) in ms }\end{array}$ & $\begin{array}{c}\text { Error of the RT } \\
\text { Variance in ms }\end{array}$ \\
\hline Patient 1 rec & $0 \%$ & $0 \%$ & 360 & 9.4 \\
\hline Patient 2 rec & $3 \%$ & $0 \%$ & 387 & 11.9 \\
\hline Healthy controls & $3.4 \%$ & $0.5 \%$ & 403 & 7.9 \\
\hline p-value for 1 rec & 0.70 & 0.56 & 0.66 & 0.64 \\
\hline
\end{tabular}

EEG spectra. A clear frontal alpha asymmetry ( $>70 \%)$ was found for alpha at F7-F8 electrodes. A peak in alpha frequency band (around $9.5 \mathrm{~Hz}$ ) was observed at F7, but not at F8 (Fig. $5 a)$. In addition, in the EEG spectra in all 3 conditions (EO, EC, GO/NOGO task) an excess of the parietal alpha rhythm (around $11.5 \mathrm{~Hz}$ ) was found at $\mathrm{Pz}$ (Fig. 5a).

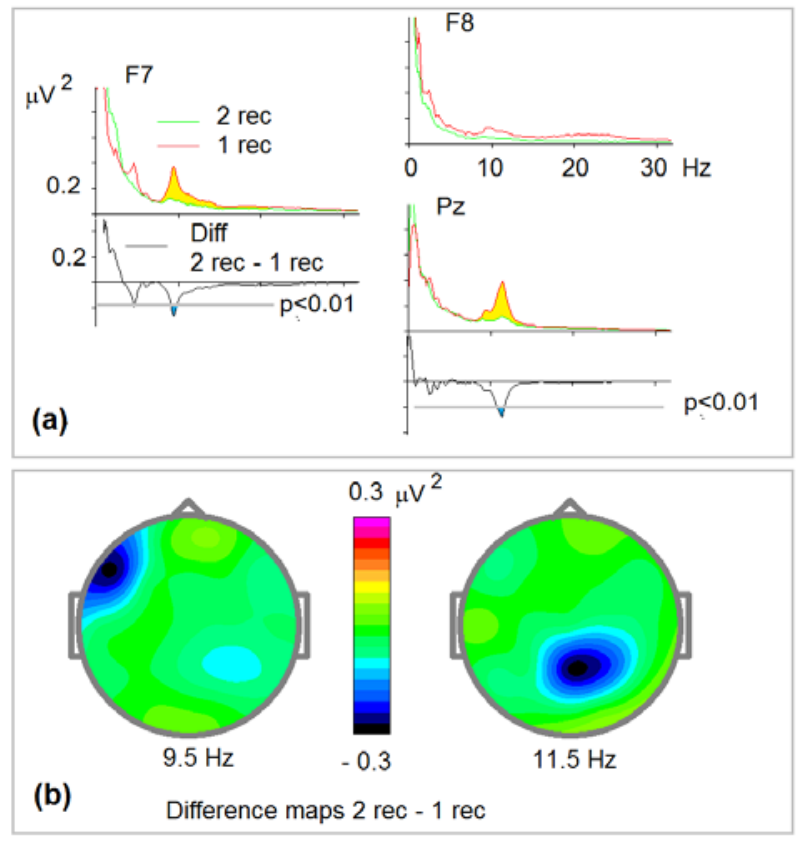

Figure 5. EEG spectra in the task condition for 1st and 2nd recordings and their differences after neurofeedback sessions; (a) EEG spectra for F7, F8 and Pz for the first recording (red), for the $2^{\text {nd }}$ recording (green) and the difference wave ( 2 rec 1 rec). Note significant $(p<0.01)$ decrease in alpha frequency band; (b) difference spectra at alpha frequency band

Event-related potentials (ERPs). Fig. 6 shows ERP for the first recording (Pre) in response to the first presentation of animal in the trials. Note excessive amplitude of $\mathrm{P} 1$ wave in comparison to the reference.

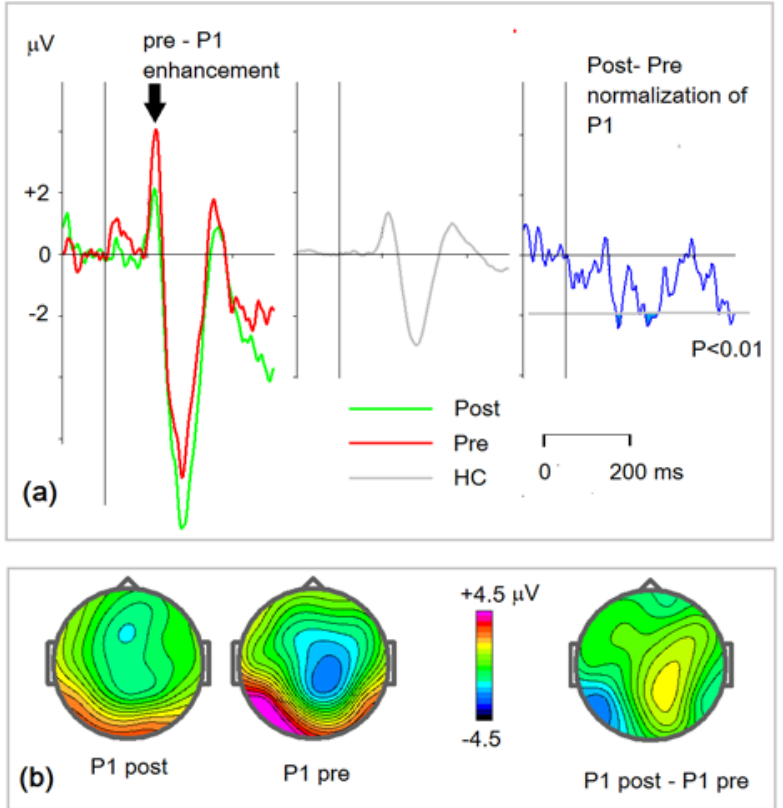

Figure 6. P1 as neuromarker of anxiety; (a) left to right: ERPs for the patient at T5 for presentation of animal at the first position in the trial: green - post, red - pre. The corresponding grand average ERP for the healthy control group (HC). Post- pre-difference wave. Confidence level $\mathrm{p}<0.01$; (b) P1 latency (120 ms) for post- pre- and the difference

Protocol of treatment - Protocol of tDCS. The asymmetrical access of alpha rhythm over the left frontal electrodes indicates inhibition (idling) of this part of the cortex in the 
patient. A similar pattern is seen in a sub-group of patients with depression [9]. Anodal tDCS of the left frontal cortex was suggested as effective treatment of this condition [12]. Following this experience, anodal tDCS at F7 was suggested for this particular patient.

Protocol of neurofeedback. As far as neurofeedback is concerned, a frontal alpha asymmetry protocol has been suggested for a subgroup of patients with asymmetrical alpha rhythms at frontal electrodes [13]. Following this experience, a neurofeedback protocol of suppression alpha rhythmicity at F7-F8 montage was suggested for this patient. The EEG spectra and a fragment of EEG in this montage during eyes open condition are shown in Fig. 7.

Alpha suppression at F7-F8 montage.

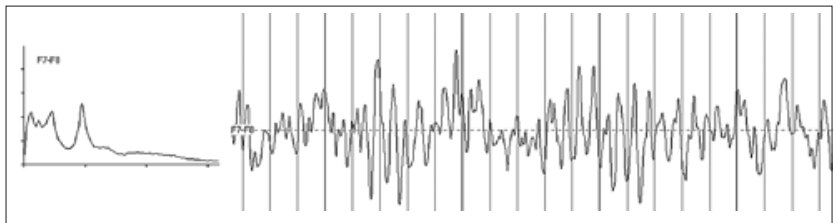

Figure 7. Neurofeedback protocol. Left - EEG spectra in F7-F8 montage. Right - a fragment of EEG in eyes open condition in F7-F8 montage. The protocol was defined as suppression of alpha frequency band at F7-F8 recording

Post-treatment assessment. Remarkable changes were observed at post- pre-difference waves both in EEG spectra and ERPs.

EEG spectra. Fig. 5 shows statistically significant changes in EEG spectra induced by the combined tDCS/neurofeedback protocol. In line with expectation, a suppression of the alpha rhythm at F7 was observed. Unexpectedly, however, suppression of the parietal alpha rhythm was also found which might indicate a general activation of the cortex induced by the tDCS/neurofeedback protocol.

ERPs. Figure 6 shows statistically significant changes in ERPs induced by the combined tDCS/neurofeedback protocol. As expected, activation of the left pre-frontal cortex led to deactivation of the amygdale which, in turn, is accompanied by suppression of the visual P1 wave.

\section{DISCUSSION}

There is abundant evidence showing lateralization of negative and positive emotions in the lateral prefrontal cortex. The idea of lateralization of the affective system first originated from the clinical observation that lesions in the left pre-frontal cortex are more often associated with induced depression, compared with lesions in the right side. In a study of Morris et al., [14] patients with lesions involving left hemisphere prefrontal or basal ganglia structures had a higher frequency of depressive disorder (75\%) than other left hemisphere lesions $(8 \%)$, or those with right hemisphere lesions.

In mental health, it has been suggested that frontal alpha asymmetry derived from quantitative electroencephalography (QEEG) may serve as a biomarker for depression. The idea is inspired by Richard Davidson's [15] theory regarding asymmetrical involvement of the orbito-frontal cortex in emotional reactions. Basically, according to this theory, the left hemisphere is biased to processing positive emotional stimuli, while the right hemisphere processes mostly negative emotional stimuli. Further research has suggested that the frontal alpha asymmetry in a resting state reflects a traitlike asymmetry of anterior cortical activity, which predicts individual differences in affective/motivational behaviours.

In the presented study, a clear frontal alpha asymmetry was observed in the patient in the first recording. This observation, in combination with existing literature of alpha asymmetry in depression, enabled the construction of an individually-tailored tDCS/neurofeedback protocol. The protocol was designed to activate the left prefrontal cortex and to reduce the frontal alpha asymmetry.

The results of the after-treatment recording were in line with expectations - the alpha at F7 was suppressed and asymmetry had disappeared. The patient recovered, and the symptoms of post-surgical depression passed. The patient claimed that she took pleasure in life again and returned to her job as a professional teacher, with which she coped splendidly.

Finally, the ERP data at the first recording showed the enhancement of P1 wave in the visual ERPs. The enhancement of P1 wave could be interpreted as a feedback effect of excessively- activated amygdala on first states of visual processing [16]. As shown in numerous studies, the amygdala, a nucleus that lies deep inside the anterio-inferior region of the medial temporal lobe, is a structure detecting threat or potential punishment, and thus generating negative emotions, such as fear and anxiety. The theory also presumes an inhibitory effect of the left prefrontal cortex on amygdala. Therefore, theoretically, it is to be expected that in the presented study activation of the left pre-frontal areas by the tDCS/neurofeedback protocol should suppress the amygdale, and respectively lead to decrease of P1 wave. Which is exactly what was observed in the ERP difference waves induced by the treatment of the presented patient!

These results mean that the symptoms of post-surgical depression might be treated with the use of the proposed methodology [17]. As proved in another study by the authors $[18,19,20]$, as in this case, the patient was able to returned to work with an improved quality of life.

\section{CONCLUSIONS}

The patient recovered from post-operative depression and returned to work after 20 sessions of the combined neurofeedback/tDCS protocol. Specific patterns of QEEG and ERPs serve as neuromarkers for constructing the protocol and for monitoring the results of intervention.

\section{REFERENCES}

1. World Health Organization. World Cancer Report 2008. International Agency for Research on Cancer, Lyon. 2009.

2. Pachalska M, Kaczmarek BLJ, Kropotov JD. Neuropsychologia kliniczna: od teorii do praktyki. Warszawa: Wydawnictwo Naukowe PWN. 2014 (in Polish).

3. Aldecoa C, Pico S, Rico J, Vazquez B, Gomez L, Garcia-Bernardo C, Gomez-Herreras J. Post-traumatic stress disorder after surgical ICU admission. Crit Care. 2010; 14 (Suppl 1): P441.

4. Luini A, Gatti G, Zurrida S, Talakhadze N, Brenelli F, Gilardi D, et al. The evolution of the conservative approach to breast cancer. Breast. $2007 ; 16: 120-9$. 
5. Meda N, Bognounou V, Seni E, Daboue A, Sanfo O. Cataract in Burkina Faso: Factors of choice between modern and traditional surgical procedures. Med Trop. 2005; 65(5): 473-6.

6. Commins DL, Atkinson RD, Burnett ME. Review of Meningioma Histopathology. Neurosurg Focus. 2007; 23(4):E3.

7. Gold C, Fachner J, Erkkilä J. Validity and reliability of electroencephalographic frontal alpha asymmetry and frontal midline theta as biomarkers for depression. Scand J Psychol. 2013; 54(2): 118 126.

8. Kropotov JD. Quantitative EEG, event related potentials and neurotherapy. San Diego: Academic Press, Elsevier, 2009.

9. Jesulola E, Sharpley CF, Bitsika V, Agnew LL, Wilson P. Frontal alpha asymmetry as a pathway to behavioural withdrawal in depression: Research findings and issues. Behav Brain Res. 2015 Jun 5; 292: 56-67. doi: 10.1016/j.bbr.2015.05.058.

10. Weinberg A, Hajcak G. Electrocortical evidence for vigilanceavoidance in Generalized Anxiety Disorder. Psychophysiology. 2011 Jun;48(6):842-51. doi: 10.1111/j.1469-8986.2010.01149.x.

11. Morel S, George N, Foucher A, Chammat M, Dubal S. ERP evidence for an early emotional bias towards happy faces in trait anxiety. Biol Psychol. 2014 May; 99: 183-92. doi: 10.1016/j.biopsycho.2014.03.011.

12. Arul-Anandam AP, Loo C. Transcranial direct current stimulation: a new tool for the treatment of depression? J Affect Disord. 2009 Oct; 117(3): 137-45. doi: 10.1016/j.jad.2009.01.016.

13. Dias AM, van Deusen A. A new neurofeedback protocol for depression. Span J Psychol. 2011 May; 14(1): 374-84. Review. PubMed PMID: 21568194.
14. Morris PL, Robinson RG, Raphael B, Hopwood MJ. Lesion location and poststroke depression. J Neuropsychiatry Clin Neurosci. 1996; 8: 399-403.

15. Davidson RJ. Anterior electrophysiological asymmetries, emotion, and depression: conceptual and methodological conundrums. Psychophysiology. 1998 Sep; 35(5): 607-14.

16. Sugase Y, Yamane S, Ueno S, Kawano K. Global and fine information coded by single neurons in the temporal visual cortex. Nature 1996; 400(6747): 869-873.

17. Graczyk M, Pąchalska M, Ziółkowski A, Mańko G, Łukaszewska B, Kochanowicz K, Mirski A, Kropotov ID. Neurofeedback training for peak performance. Ann Agric Environ Med. 2014; 21(4): 871-875. doi: $10.5604 / 12321966.1129950$.

18. Chantsoulis M, Mirski A, Rasmus A, Kropotov JuD, Pachalska M. Neuropsychological rehabilitation for traumatic brain injury patients. Ann Agric Environ Med. 2015; 22(2): 368-379. doi: 10.5604/12321966.1152097.

19. Pąchalska M, Kropotov ID, Mańko G, Lipowska M, Rasmus A, Łukaszewska B, Bogdanowicz M, Mirski A. Evaluation of a neurotherapy program for a child with ADHD with Benign Partial Epilepsy with Rolandic Spikes (BPERS) using event-related potentials. Med Sci Monit. 2012; 18(11): CS94-104.

20. Chrapusta A, Pąchalska M, Wach R. Evaluation of the health-related quality of life during the treatment of severe burn complicated by multiple organ dysfunction syndrome (MODS). Acta Neuropsychologica 2014; $12(4)$ : 483-492. 